\title{
Thermomechanical Testing Machine Conceived for the Study of Shape Memory Alloys. Application to the Training and Testing of the Two-Way Memory Effect
}

\author{
A. Villafaña*, M. Masse ${ }^{* * *}$, R. Portier**** and J. Pons*** \\ * Laboratoire de Métallurgie Structurale, ENSCP, 11 rue Pierre et Marie Curie, 75231 Paris cedex 05, \\ France \\ ** CECM/CNRS, 15 rue G. Urbain, 94400 Vitry-sur-Seine, France \\ *** Dept. Fisica, Universitat de les Illes Balears, 07071 Palma de Mallorca, Spain
}

\begin{abstract}
An electrohydraulic testing machine dedicated to the study of shape memory alloys has been developed. A complete computer control of force (resolution $\pm 1 \mathrm{~N}$ ), elongation (sensitivity $\pm 10 \mu \mathrm{m}$ ) and temperature (precision $1 \mathrm{~K}$ ) has been built. The machine is able to work in a soft mode, i.e. force controlled, or a hard mode i.e. deformation controlled and in a range of temperatures from $173 \mathrm{~K}$ to $473 \mathrm{~K}$.

The performances of the system have been checked by studying the two-way shape memory effect induced by thermomechanical cycling in $\mathrm{Cu}-\mathrm{Zn}-\mathrm{Al}$ alloys. The training cycles consist of cooling down to the $\mathrm{Mf}$ temperature under a uniaxial tensile stress of $35 \mathrm{MPa}$ followed by heating up to Af at zero stress. The cycling procedures are completely automatic. A comparison of the two-way memory effect in single crystals (nominal Ms of $273 \mathrm{~K}$ ) and polycrystals (nominal Ms of $230 \mathrm{~K}$ ) is reported.

A study in compression of tensile educated crystals shows the aptitude of these specimens to produce work.
\end{abstract}

\section{INTRODUCTION}

Shape memory alloys (SMA) require particularly precise thermomechanical testing and training procedures. These procedures may in addition vary considerably from experiment to experiment depending on the properties being researched. This combination of precision and flexibility is seldom met by commercially available testing machines. Most conventional tensile testing machines working on a hard mode for example are unable to compensate for grip thermal expansion and have led in the past to unsuitable training procedures (for example the so-called "combined" training routine [1]). This has made it difficult to estimate the contribution of such types of training to the microstructural asymmetry required to produce the TWME.

This explains the effort made by some researchers on building their own testing prototypes designed to avoid thermal dilatation problems. Unfortunately, most of these prototypes make use of non-uniform deformation modes (torsion or bending training routines) to amplify the two way memory displacement with respect to thermal dilatation $[2,3]$. However, the use of these deformation modes makes the analysis of strain and stress particularly complicated.

\section{EXPERIMENTAL}

\subsection{The thermomechanical testing machine}

Instead of struggling with a theoretical assessment of the training parameters for the case of non-homogeneous deformation modes, we have preferred to build a new testing apparatus bearing in mind to minimise the known contributions of instrumental error presented by conventional testing machines. These errors are, as mentioned, mostly of thermal origin and consist in thermal dilatations of mechanical structures of a testing machine or sensors working in inadequate conditions. Other sources of error are noisy or unstable signals. In order to optimise at maximum the stress, strain and temperature control, the working ranges of values of these parameters were limited to those usually required for the SMA testing. The result is a uniaxial strain or stress controlled testing machine schematically shown in figure 1 [4]. The instrument is electrohydraulic-powered, a motor pump feeds a hydraulic piston with 210 bar oil pressure. The force applied by the piston is regulated by a servovalve steered by an analog control electronic. Finally, a microcomputer acting on the latter by means of two data acquisition cards allows for a versatile programming of any thermomechanical test (composed by the desired number of steps), 


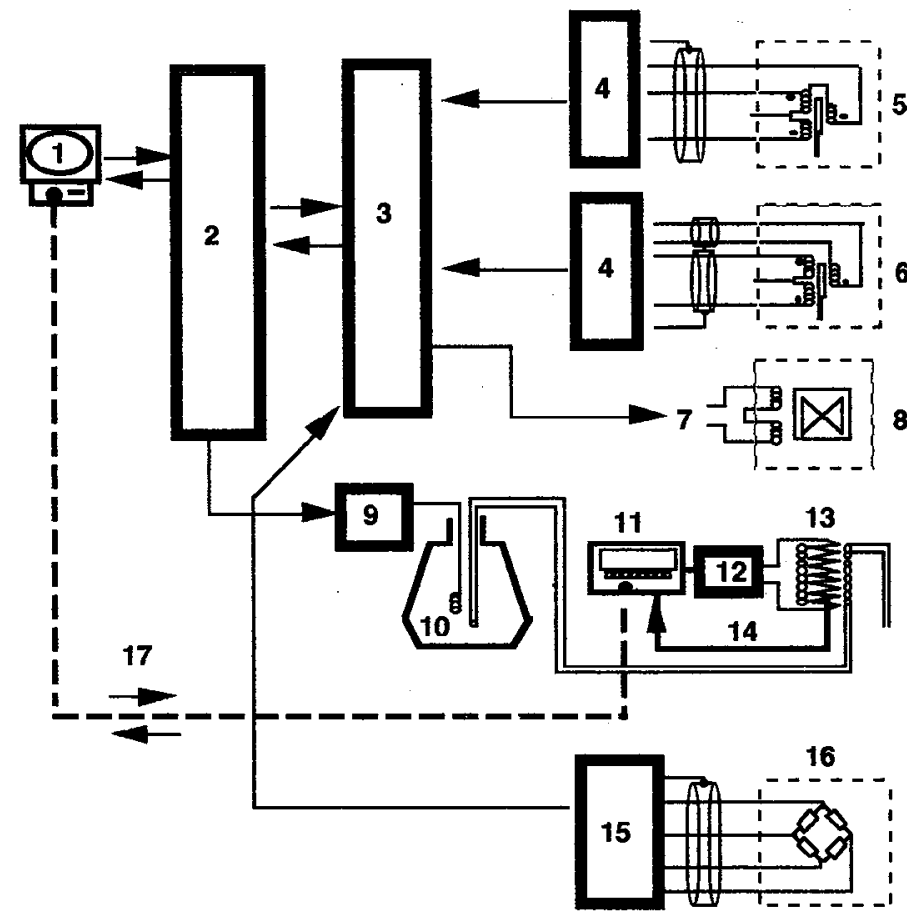

1 Macintosh microcomputer

2 D/A A/D Interface

3 Analog control electronic

4 Position signal conditioning

5 1st position sensor (LVDT)

6 2nd position sensor (LVDT)

7 Hydraulic servovalve

8 Hydraulic piston

9 N2 evaporation control relay

10 Liquid nitrogen evaporator

11 Temperature controller

12 Cryofurnace power unit

13 Cryofurnace

14 Thermocouple

15 Force signal conditioning

16 Load cell

17 RS-232 serial communication

Fig. 1 - Schematic representation of the thermomechanical testing machine.

surveying the overall execution of the experiment and recording the acquired data. The data acquisition cards are composed by 12 and 16 bit multiple channel A/D D/A converters and several digital I/O lines. For the computer programming, a high flexibility is obtained by the use of a dedicated instrumentation software package (National Instruments, Labview), by means of which a set of programs specially adapted for SMA education protocols has been developed.

A particular care was devoted to prevent electromagnetic noise from distorting sensor output signals (all signal wires were properly shielded). In spite of that, a closer inspection showed that the low-level load cell output was particularly sensitive to electromagnetic noise sources such as power lines. The worst case was found when the cryofurnace was tested at its maximum heating activity. The load cell signal is represented in figure 2 left, in its as-sampled condition. This figure shows the simple case of a constant load signal with no furnace activity sampled over $1.5 \mathrm{~s}$ time intervals at a rate of $40 \mathrm{KHz}$. As can be seen, the amplitude of the ordinary electromagnetic noise is approximately ten times greater than the D/A converter resolution. That means a loss of precision of the force signal of one order which in our case corresponds to $2,2 \mathrm{~N}$. The right graph in figure 2 shows the numerically low-pass filtered signal. An analysis of the frequency components of the signal in this case has permitted to attribute the origin of the electromagnetic noise to the power lines. Another important factor of inaccuracy in the measurement of the force signal was found in connection with the cryofurnace working at full power: The measured values gradually deviated from the ideal measure as the load cell temperature varied. This effect is shown in figure 3 for the case of a typical force magnitude of $30 \mathrm{dN}$ used in the thermomechanical cycling protocols described further in this paper. It is important to remember that in a closed control loop it is not possible to verify that the recorded measures correspond to the physical reality. Hence, a careful reproduction of the actual experimental conditions via an open control loop was realised to ensure the reliability of the measurement. Finally, a thermal stabilisation device was designed for the stainless steel shaft transmitting the effort to the load cell in order to minimise thermal gradients in the load cell. As a result, the precision of the force measurements was substantially improved as shown in figure 4 for the case of a specimen subjected to the thermal conditions of a training protocol. In this experiment the specimen's temperature varied at a rate of $4 \mathrm{~K} \mathrm{~min}^{-1}$ but no stress was applied. The significant technical features of the instrument can be summarised as follows : 
Signal brut (binaire)

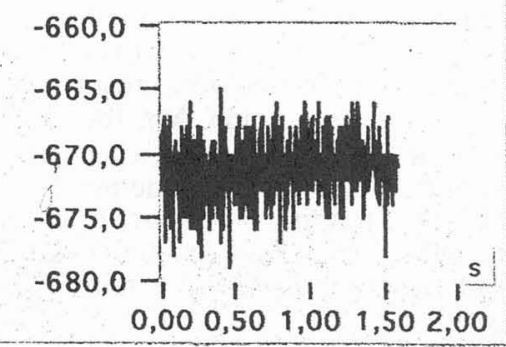

Signal filtré

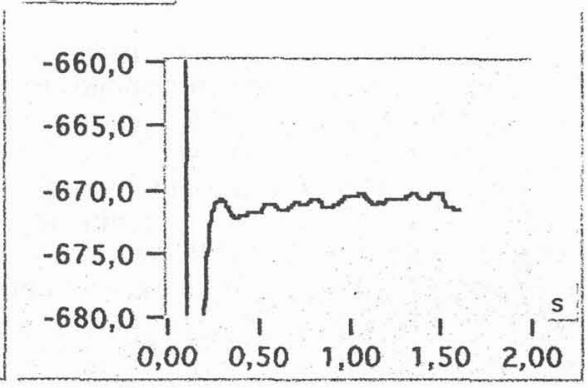

Fig. 2 - Force signal measurements, left: as sampled signal; right: after numerical filtering.

Operation modes: force controlled (soft mode) and strain controlled (hard mode). The default mode is the soft mode which is particularly useful to avoid undesirable straining of the specimen while mounting it.

The force, elongation and temperature working ranges and their maximum rates are :

$$
\begin{array}{ll}
\mathrm{F}[-500 ; 5000] \pm 1 \mathrm{~N} & |\mathrm{dF} / \mathrm{dt}| \leq 100 \mathrm{~N} \mathrm{~s}^{-1} \\
\Delta 1[-2.5 ;+2.5] \pm 0.01 \mathrm{~mm} & |\mathrm{~d} \Delta \mathrm{d} / \mathrm{dt}| \leq 10 \mathrm{~mm} \mathrm{~s}^{-1} \\
\mathrm{~T}[173 ; 473] \pm 0.5 \mathrm{~K} & \left|\mathrm{dT} / \mathrm{dt}_{\text {cryofurnace }}\right| \leq 5 \mathrm{~K} \cdot \mathrm{min}^{-1}
\end{array}
$$

The controlled parameters are applied in the form of ramps and dwells and can be programmed in almost every combination or sequence within the mentioned boundaries. A wide variety of thermomechanical tests is thus possible. It is interesting to point out, for example, that a strict control of the applied stress on the specimen allows to carry out a dilatometer-like test without removing the sample.

\subsection{Studied alloys}

In order to qualify the testing machine a TWME study on copper-based SMA has been carried out. Three $\mathrm{Cu}-\mathrm{Zn}$-Al samples, two of them polycrystalline and a single crystal were studied. The samples are cylindrical $\emptyset=3 \mathrm{~mm}$, with gauge lengths between 10 and $20 \mathrm{~mm}$ and threaded heads. The polycrystalline samples have a composition $\mathrm{Cu}-15.8 \mathrm{wt} . \% \mathrm{Zn}-8.3 \mathrm{wt} \% \mathrm{Al}$, nominal $\mathrm{M}_{\mathrm{S}}=230 \mathrm{~K}$, and a mean grain size of $1 \mathrm{~mm}$. The single crystal is a $\mathrm{Cu}-16.9 \mathrm{wt} . \% \mathrm{Zn}-7.7 \mathrm{wt} . \% \mathrm{Al}$ with nominal $\mathrm{M}_{\mathrm{S}}=273 \mathrm{~K}$. All three samples were spark machined and subsequently heat treated. The heat treatment consisted in annealing at $1123 \mathrm{~K}$ for $1.8 \mathrm{Ks}$ and air-cooling.

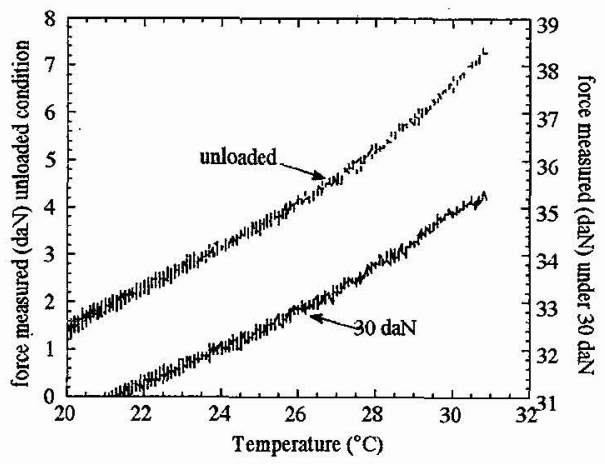

Fig. 3 - Comparison of the measured force with varying temperature of the load cell corresponding to a worst-case testing condition.

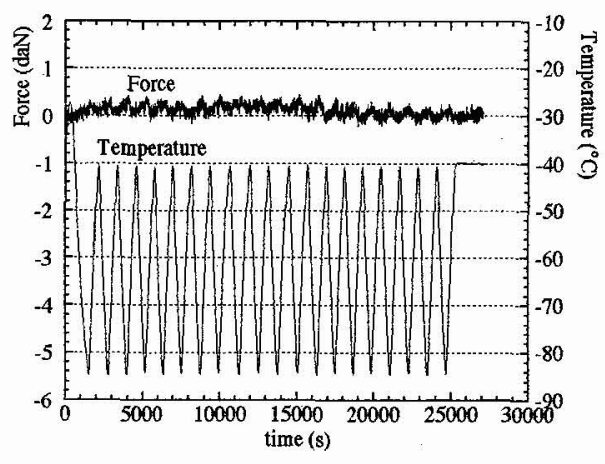

Fig. 4 - Recorded derive of the force measure from its ideal value (zero $\mathrm{N}$ ) after thermal stabilisation and isolation of structural parts between the load cell and the cryofurnace. 


\subsection{Thermomechanical cycling}

Two different thermomechanical cycling (training) protocols have been employed with the polycrystalline specimens. The first one corresponds to the training procedure employed by Stalmans et al [5]. In this procedure, a stress is applied to the sample being at an initial temperature above $A_{f}$, then the sample is cooled (while keeping the stress constant) to a temperature below $\mathbf{M}_{\mathrm{f}}$, the sample is then unloaded and it is finally reheated to the initial temperature. It follows a thermal cycle (the sample is cooled and reheated as in the training cycle but without any applied stresses). The thermal cycles serve to follow the evolution of the acquired TWME throughout the whole training procedure. In all experiments a tensile training stress of $35 \mathrm{MPa}$ was used. Such a training procedure will be denoted "1/1" hereafter. In the second procedure one thermal cycle is carried out only after twenty consecutive training cycles. Such a training protocol will be denoted "20/1". A variation of this protocol can be for example a "20/2" sequence where two thermal cycles instead of one are performed. The aim of using the second training procedure can be rationalised as follows: it is rather well accepted that the two-way shape memory effect obtained after thermomechanical training is related to the formation of properly oriented dislocation structures during training, which favour the nucleation and growth of the trained martensitic variants [2, $3,5]$. During stress-free thermal cycling, however, a distribution of differently oriented dislocations is formed $[6,7]$. So, during the $1 / 1$ training protocol, in addition to the well oriented dislocations favouring the trained variants, other dislocations can be formed which do not assist the TWME or can even be detrimental for it. In this sense, a poorer TWME is expected to be obtained by the $1 / 1$ training procedure, specially during the first cycles, when many variants different than the trained ones can grow during the stress-free thermal cycles and create unfavourably oriented dislocations.

Finally, the behaviour of the fully trained specimens thermally transforming under an opposing compressive stress was studied in order to check the ability of the material to perform work on cooling.

\section{RESULTS AND DISCUSSION}

\subsection{The $1 / 1$ training of polycrystalline specimens}

The figures 5 and 6 show the resulting evolution of the TWME in polycrystalline specimens using the 1/1 training method. The figure 5 shows the elongation of the specimen (in \%) as recorded in function of the time for the first 33 couples of $1 / 1$ education-and-training cycles. The effect of the training cycles can be clearly distinguished from that of the thermal cycles by the much higher elongation resulting from a transformation under stress. The training cycles reach a maximum

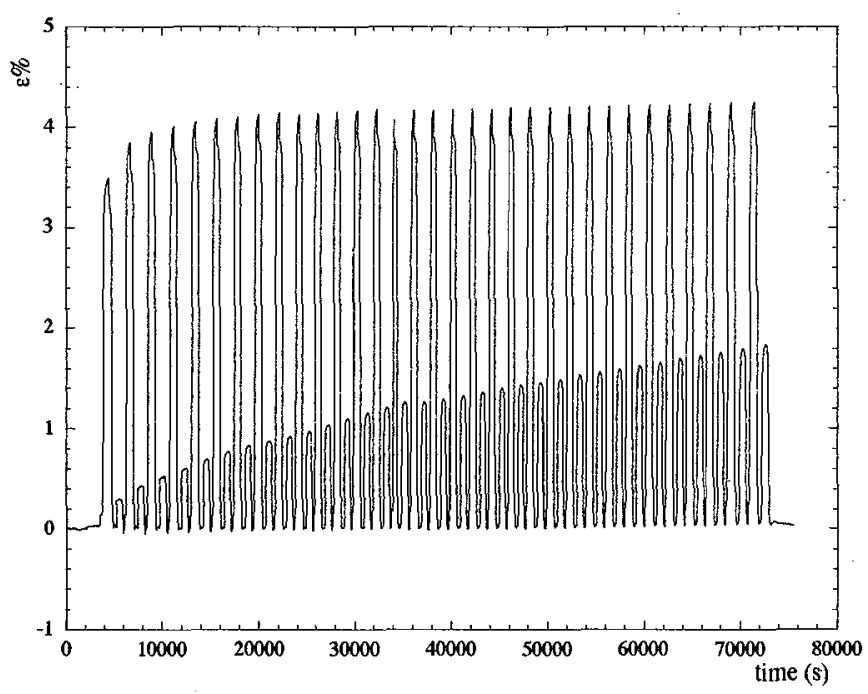

Fig. 5 - Thermomechanical cycling (education) of a polycrystalline sample. First 33 cycles performed with a $1 / 1$ education protocol elongation due to the martensitic transformation under stress (about $3.6 \%$ for the first training cycle), but this elongation also includes the elastic deformation of the martensite. When the transformation is complete and the specimen is released from the training stress, a somewhat lower elongation remains $(3.2 \%$ for the first training cycle). This remaining elongation, known as stress assisted transformation effect (SATE), is also a characterising parameter of the thermomechanical cycling training process. In the depicted training process it can be observed that the SATE evolves rapidly during the first four cycles but then saturates at about $4 \%$ elongation. Concerning the TWME elongation recorded during the thermal cycles, it presents a more regular evolution growing steadily from $0.3 \%$ for the first thermal cycle 


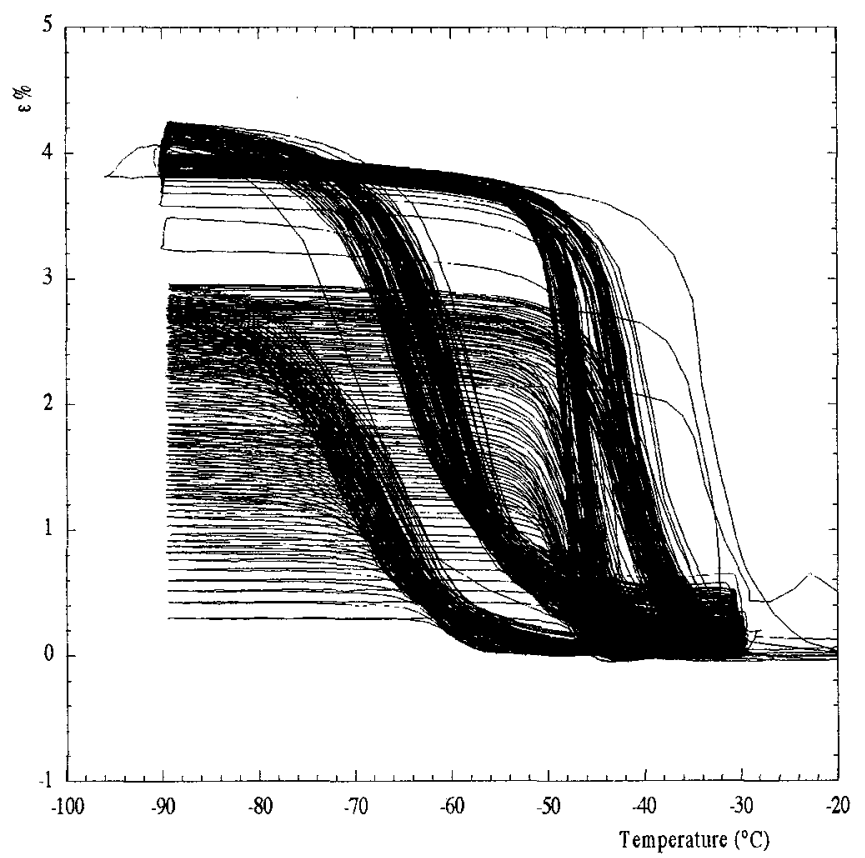

Fig. 6 - Thermomechanical cycling of a polycrystalline sample. Elongation (\%) in function of the temperature. "Complete education" after $1001 / 1$ couples.

to $1.8 \%$ for the 33 rd thermal cycle. The figure 6 is a elongation versus temperature representation of the whole thermomechanical cycling process which consisted in $1001 / 1$ couples. This representation gives a more accurate information about the way the transformation proceeds within each cycle. The regularity of the evolution of the TWME during the entire experiment is evident. The elongation associated with the TWME reaches nearly $3 \%$ with the 100 th thermal cycle. It can be also noted that the SATE remains at the $4 \%$ value already obtained during the first cycles (fig. 5). When in the $n^{\text {th }}$ $1 / 1$ cycle the specimen is reheated to the temperature above $A_{f}$, the high temperature form is mostly recovered. However, a closer examination reveals that a very small deformation remains unrecovered with respect to the original length before the cycling. This so-called residual deformation (RD) is associated with retained martensite and has been reported to cumulate with the number of thermomechanical cycles performed [5]. It is generally assumed that this RD disappears if the specimen is reheated to a temperature substantially higher than Af. The training experiment formerly described resulted in a relatively sniall RD.

\subsection{The $20 / 2$ training protocol}

It is clear from the above that the parameters SATE, TWME and RD are helpful to describe the specimen's behaviour during training and will therefore be used in the following for a concise description of two other training experiments: The alternative 20/2 training protocol was first employed with a polycrystalline specimen, and then with the single crystal sample. Both results are summarised in figures

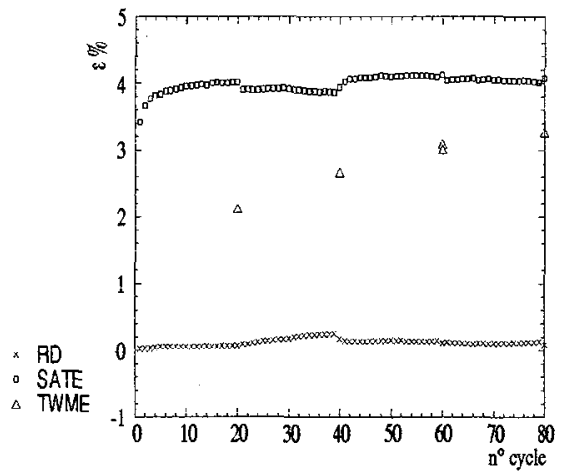

Fig. 7 - The SATE, TWME and RD evolution with a polycrystalline specimen subjected to a 20/2 training protocol (TWME is tested only every 20 cycles).

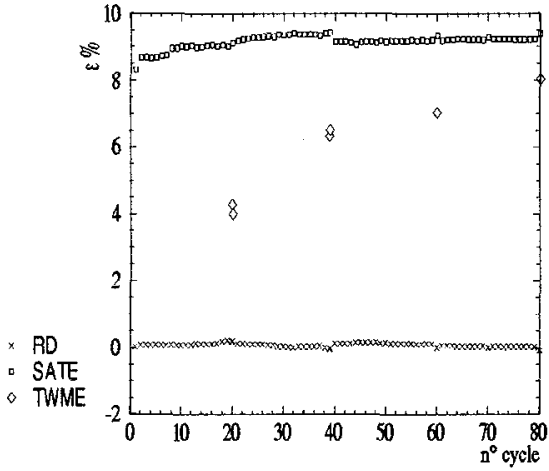

Fig. 8 - The SATE, TWME and RD behaviour in the case of the single crystal specimen using the same 20/2 thermomechanical cycling procedure. 


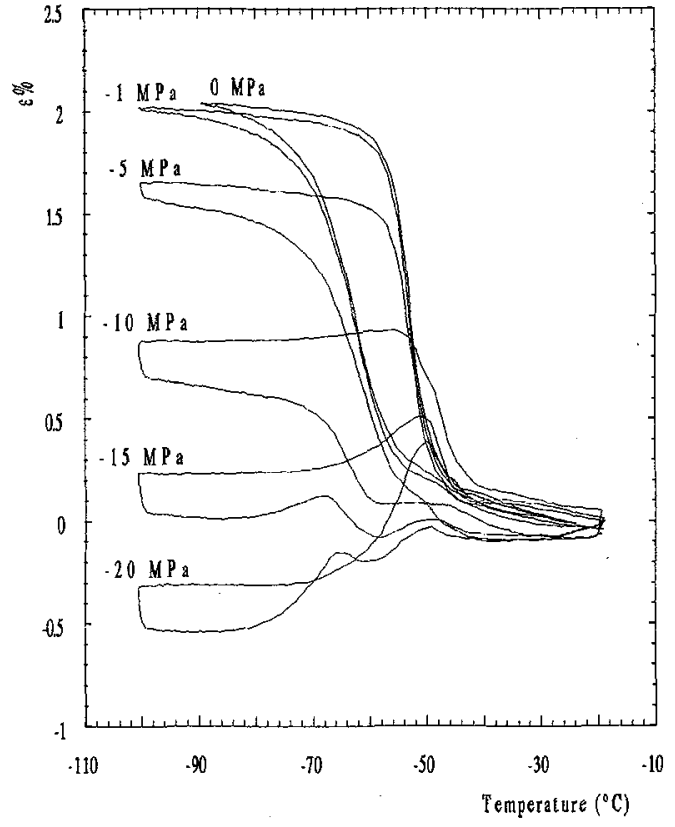

Fig. 9 - Behaviour of a tensile-trained polycrystalline specimen subjected to different compressive stresses. The sample was thermomechanically cycled using a $1 / 1$ protocol.

7 and 8 respectively. From figure 7 it can be concluded that the $20 / 2$ protocol leads to different results than the 1/1. A somewhat better TWME of $3.3 \%$, a similar SATE of $4 \%$ and a weak RD are observed. However, the same TWME is more rapidly obtained by using the alternative method. A comparison of the 20 th cycle in both methods shows a $2.2 \%$ for the $20 / 2$ protocol against only $1.4 \%$ for a $1 / 1$ protocol. This results show the detrimental effect of the dislocations introduced by thermal cycling, as commented above.

The results obtained with the single crystal specimen after $80(20 / 2)$ cycles are a TWME of $8 \%$, a SATE of $9 \%$ and a weak RD. The comparatively much greater SATE and consequently greater TWME of the single crystal specimen with respect to the polycrystalline samples are due to the fact that such a specimen undergoes a mostly monovariant martensitic transformation. This is not the case of polycrystalline samples where the stress concentration in grain boundaries promotes the appearance of other crystallographic variants.

\subsection{Compression of tensile trained samples}

The behaviour of tensile trained SMA specimens subjected to compressive static stresses was investigated. The figure 9 shows the case of a polycrystalline sample working against compressive stresses going from -1 to $-20 \mathrm{MPa}$ during transformation. This sample had been trained using the $1 / 1$ protocol for 100 couples. To perform the compression test a stress was applied to the trained sample at a temperature above $A f$ then the sample was cooled to a temperature below Af, the stress was then released and finally, the sample was reheated to the initial temperature. The graph shows that during cooling the sample continues to produce elongations going in the sense of the training stress until an opposing stress of $15 \mathrm{MPa}$ is applied. At this opposing stress level, every spontaneous attempt of the specimen to produce an elongation is essentially neutralised. For higher stresses a contraction of the sample is produced. A similar test was performed with the polycristalline specimen trained with the 20/2 protocol as described in figure 7 . Approximately the same opposing stress of $15 \mathrm{MPa}$ was necessary to hinder any elongation of the specimen.

\section{CONCLUSIONS}

The quality of the experimental data obtained by the use of this apparatus proved to be satisfactory. Some sources of instrumental errors were identified and minimised in order approach the ideal experimental conditions required to properly define and record the thermomechanical story of each studied sample. Comparative SMA training experiments with polycrystalline and single crystal samples have shown the possibilities offered by this apparatus to study the properties of these alloys. Once the capacities of the instrument proved, further work is being developed on the TWME subject.

\section{References}

[1] Perkins J., in Shape Memory Alloy '86 (C. Yougi Ed.) China Academic, Guilin (1986) p. 201.

[2] Rodriguez P., Thèse de doctorat, INSA Lyon, (1989).

[3] Contardo L., Guénin G., Acta Metall. Mater, 38 (1990) p. 1267.

[4] Villafaña A., Thèse de doctorat, Univ. P. et M. Curie (Paris 6) (1997).

[5] Stalmans R., Van Humbeeck J. and Delaey L.: Acta metall. mater. 40 (1992) pp. 501-511, 2921-2931.

[6] Pons J., Lovey F.C. and Cesari E. 1990 : Acta Metall. Mater. 38, p. 2733.

[7] Amengual A., Cesari E. and Pons J. 1995 : J. Physique IV, Colloque C8, Vol. 5 (1995) p. 871. 